\title{
Orders on Multisets and Discrete Cones
}

\author{
Marston CONDER \\ Department of Mathematics, University of Auckland \\ m. conder@auckland.ac.nz \\ Simon Marshall \\ Department of Mathematics, Princeton University \\ slm@math.princeton.edu \\ ArKadil SlinKo* \\ Department of Mathematics, University of Auckland \\ a.slinko@auckland.ac.nz
}

\begin{abstract}
We study additive representability of orders on multisets (of size $k$ drawn from a set of size $n$ ) which satisfy the condition of Independence of Equal Submultisets (IES) introduced by Sertel and Slinko (2002, 2007). Here we take a geometric view of those orders, and relate them to certain combinatorial objects which we call discrete cones. Following Fishburn (1996) and Conder and Slinko (2004), we define functions $f(n, k)$ and $g(n, k)$ which measure the maximal possible deviation of an arbitrary order satisfying the IES and an arbitrary almost representable order satisfying the IES, respectively, from a representable order. We prove that $g(n, k)=n-1$ whenever $n \geq 3$ and $(n, k) \neq(5,2)$. In the exceptional case, $g(5,2)=3$. We also prove that $g(n, k) \leq f(n, k) \leq n$ and establish that for small $n$ and $k$ the functions $g(n, k)$ and $f(n, k)$ coincide.
\end{abstract}

AMS classification: 06F25, 91B16

Keywords: multiset, linear order, additive representability, Independence of Equal Submultisets

* Corresponding author. 


\section{Introduction}

One of the most common preference orderings in economics is that of bundles of commodities, and one of the most common problems is to rationalise such an ordering, representing it by a numerical order-preserving function of utilities of those commodities. The space of commodity bundles can be naturally represented as a subset of a Cartesian product $X=\prod_{i=1}^{n} X_{i}$, where $X_{i}$ can vary depending on the model.

It is often assumed that each $X_{i}$ has a very rich structure and is isomorphic to a subset of $\mathbb{R}$, so that $X$ becomes a subset of a finite-dimensional Euclidean space $\mathbb{R}^{n}$. This model corresponds to existence of $n$ types of infinitely divisible goods. An element $\mathbf{x}=\left(x_{1}, \ldots, x_{n}\right) \in X$ denotes a bundle with $n$ goods, and $x_{i}$ shows the quantity of the $i$ th good. This case was extensively studied by J. von Neumann and O. Morgenstern [?], J. Marshak [?], I.N. Herstein and J. Milnor [?] and others.

Another extreme case is when elements in each $X_{i}$ belong to the same class but cannot be compared and measured in units of something, such as when $X_{1}=\{$ apple, banana $\}$ and $X_{2}=\{$ pepsi, coca cola $\}$. In this particular case the $X_{i}$ have no structure at all and are arbitrary finite sets. This case was studied by Krantz et al [?] and significant progress was made on it by P. Fishburn [?]. The case most studied is one where every $X_{i}$ consists of just two elements. In this case elements of the Cartesian product $2^{[n]}$ can be identified with subsets of $[n]=\{1,2, \ldots, n\}$ and we obtain the classical case of comparative probability orders [?, ?] for which the basic rationality condition is the following axiom of de Finetti:

Definition 1. A linear order $\preceq$ on $2^{[n]}$ is called a comparative probability if it satisfies the following de Finetti axiom: for any $A, B \in 2^{[n]}$ and any $C \in 2^{[n]}$ such that $C \cap(A \cup B)=\emptyset$

$$
A \succeq B \Longleftrightarrow A \cup C \succeq B \cup C .
$$

There is an important intermediate case which has not been studied systematically so far. We often have $n$ types of goods which are divisible to a certain extent but not infinitely divisible (such as money, cars, houses etc.). These goods can be measured only in whole units of some quantity which is further indivisible. Let us call these goods finitely divisible. If $X_{i}$ denotes the quantity of a certain finitely divisible good, then each $X_{i}$ has the structure of the monoid of nonnegative integers $\mathbb{N}$. This model has numerous useful interpretations.

When we are in the situation of the $X_{i}$ representing finitely divisible goods, the bundle $\mathbf{x}=\left(x_{1}, \ldots, x_{n}\right) \in X$ can be replaced with the $n$-tuple of 
nonnegative integers $\left(k_{1}, \ldots, k_{n}\right)$, where $k_{i}$ is the number of indivisible units that are contained in $x_{i}$. The main motivating example for this study comes from Sertel and Kalaycıŏlu [?] and Sertel and Slinko [?, ?].

Example 1. Consider a k-member parliament or committee. There are $n$ political parties to which the members are affiliated. Here $i$ is the type of political party and $k_{i}$ is the number of members of this political party elected to the parliament. The total number of elected parliamentarians in parliament $\mathbf{x}=\left(k_{1}, \ldots, k_{n}\right)$ is $k=\sum_{i=1}^{n} k_{i}$. The relation $\mathbf{x} \succeq \mathbf{y}$ means that a voter thinks parliament $\mathbf{x}$ is at least as good as parliament $\mathbf{y}$.

Mathematically speaking, a parliament is a multiset on the set of political parties $[n]=\{1,2, \ldots, n\}$. Unlike sets, multisets allow multiple entry of elements, so a parliament can be represented as a multiset $M=$ $\left\{1^{k_{1}}, 2^{k_{2}}, \ldots, n^{k_{n}}\right\}$, where $i^{k_{i}}$ means that element $i$ (party $i$ ) enters the multiset (the parliament) $k_{i}$ times. The number $k_{i}$ is called the multiplicity of $i$ in $M$. The multiset $M$ can also be described as $([n], \mu)$ where $\mu:[n] \rightarrow \mathbb{N}$ is the multiplicity function given by $\mu(i)=k_{i}$ for all $i \in[n]$. The sum of multiplicities $k=k_{1}+k_{2}+\ldots+k_{n}$ is called the cardinality of $M$.

The set of all multisets of fixed cardinality $k$ on the set $[n]$ will be denoted by $\mathcal{P}_{k}[n]$. The set of all multisets will be denoted $\mathcal{P}[n]=\bigcup_{k=1}^{\infty} \mathcal{P}_{k}[n]$. The union $M_{1} \cup M_{2}$ of two multisets $M_{1}=\left([n], \mu_{1}\right)$ and $M_{2}=\left([n], \mu_{2}\right)$ is again a multiset on $[n]$ whose multiplicity function is defined as $\mu_{1}+\mu_{2}$. Similarly, the intersection $M_{1} \cap M_{2}$ is given by the multiplicity function $\min \left(\mu_{1}, \mu_{2}\right)$, where this minimum is defined pointwise on $[n]$. Next, we say that $M_{1}$ is a submultiset of $M_{2}$, if $\mu_{1}(i) \leq \mu_{2}(i)$ for all $i \in[n]$, and we denote this by $M_{1} \subseteq M_{2}$. For more information on multisets see, for example, [?].

Definition 2. An order $\succeq$ on $\mathcal{P}_{k}[n]$ (or on $\mathcal{P}[n]$ ) is said to be (additively) representable if there exist nonnegative real numbers $u_{1}, \ldots, u_{m}$ such that for all $M_{1}=\left([n], \mu_{1}\right)$ and $M_{2}=\left([n], \mu_{2}\right)$,

$$
M_{1} \succeq M_{2} \Longleftrightarrow \sum_{i=1}^{n} \mu_{1}(i) u_{i} \geq \sum_{i=1}^{n} \mu_{2}(i) u_{i} .
$$

We will refer to the coefficients $u_{1}, \ldots, u_{n}$ as the utilities; here the word 'utility' serves as a generic name for a number of related but distinct concepts.

Additive representability of preference orders is well-established topic of study. A systematic discussion of this idea and its history can be found in the monograph [?]. In the most studied case of comparative probability, de Finetti's axiom (??) is not sufficient for additive representability - indeed, one needs infinitely many additional cancellation conditions [?]. Because of 
this, it is quite surprising that for an order $\succeq$ on $\mathcal{P}[n]$, the multiset analogue of (??), in which the union is understood as the multiset union and the condition $C \cap(A \cup B)=\emptyset$ is dropped, is nearly sufficient for additive representability.

It was discovered by Robbiano [?] and rediscovered by Danilov [?] and Martin [?] that for any order satisfying the multiset analogue of de Finetti's axiom, there is an $m \times n$ matrix of weights, with $m \leq n$, that can be used to determine the order in which two multisets $A$ and $B$ appear in $\succeq$. The weights from the first row are used first, but if the total weights of $A$ and $B$ relative to this system of weights are the same, then the weights from the second row are used, and so on. Multisets with the multiset analogue of (??) have been extensively studied in computer science with regard to termination of rewriting systems; see [?].

We now note that there is an essential difference between this framework and Example ??. In the latter, the total number of objects $k=\sum_{i=1}^{n} k_{i}$ is fixed, making us deal with an order on $\mathcal{P}_{k}[n]$, and there is no obvious analogue of de Finetti's axiom. It is exactly the framework of Example ?? that Sertel and Slinko studied in [?, ?] and we continue to study in this article. We are interested in additive representation of orders on $\mathcal{P}_{k}[n]$. This case is markedly different from the case of orders on $\mathcal{P}[n]$, and is much more similar to the case of comparative probability, explaining why Fishburn's ideas could be made to work.

By an order we understand any reflexive, complete and transitive binary relation, which will be denoted as $\succeq$. If it is also antisymmetric, it will be called a linear order. The notation $\mathbf{x} \succ \mathbf{y}$ will mean $\mathbf{x} \succeq \mathbf{y}$ but not $\mathbf{y} \succeq \mathbf{x}$.

The problem considered here and in many similar situations (as in [?, ?, ?, ?] for example) is to impose minimal conditions on the order $\succeq$ on $\mathcal{P}_{k}[n]$ to guarantee its representability. This means that the voter must be (to a certain extent) rational in order for their preferences to be representable. The question is, just how rational they should be.

The following basic rationality condition was suggested by Sertel and Slinko [?, ?], who called it consistency. Here we give a slightly different (but equivalent) definition of this concept, which makes it a close relative to the concept of the Independence of Equal Coordinates [?, p.30].

Definition 3. An order $\succeq$ on $\mathcal{P}_{k}[n]$ is said to satisfy the Independence of Equal Submultisets condition (IES) if for every two multisets $U$ and $V$ of the same cardinality $j$ with $1 \leq j \leq k-1$, the relation $U \cup W \succeq V \cup W$ holds for one particular multiset $W$ of cardinality $k-j$ if and only if this relation holds for every multiset $W$ of cardinality $k-j$.

This is also an analogue of de Finetti's axiom for comparative probability orders [?, ?]. 
If $\succeq$ is an order on $\mathcal{P}_{k}[n]$ which satisfies the IES, then, for all $j$ in the range $1 \leq j \leq k-1$, it induces an order $\succeq_{j}$ on $\mathcal{P}_{j}[n]$, which also satisfies the IES. To define $\succeq_{j}$ we take any $W \in \mathcal{P}_{k-j}[n]$, and then for any $U, V \in \mathcal{P}_{j}[n]$, we set

$$
U \succeq_{j} V \text { if and only if } U \cup W \succeq V \cup W .
$$

By the IES, this will not depend on the choice of the particular multiset $W$. The orders $\succeq_{j}$ will be called orders associated with $\succeq$. These orders, as it was proved in Lemma 1 of [?], satisfy the following property.

Lemma 1. Let $\succeq$ be an order on $\mathcal{P}_{k}[n]$ which satisfies the IES and $\succeq_{i}$ be the corresponding order on $\mathcal{P}_{i}[n]$ for $i=1, \ldots, k$. Suppose that $U, V \in \mathcal{P}_{\ell}[n]$, $R, Q \in \mathcal{P}_{h}[n]$, and $U \succeq_{\ell} V, R \succeq_{h} Q$ with $\ell+h \leq k$. Then $U \cup R \succeq_{\ell+h} V \cup Q$.

In this paper, without loss of generality, we assume that $\succeq$ induces a linear order on $[n]$ (which is naturally identified with $\mathcal{P}_{1}[n]$ ), and that this linear order satisfies the condition

$$
1 \succ_{1} 2 \succ_{1} 3 \succ_{1} \ldots \succ_{1} n
$$

It is important that we rule out the possibility of indifferences here. This does not restrict our framework, since if $j \sim i$ for some $j>i$, then we may view the elements $i, i+1, \ldots, j$ as indistinguishable, and then we may simplify the framework by not making any distinction between these elements.

The next definition is technical but very important.

Definition 4. We say that an order $\succeq$ on $\mathcal{P}_{k}[n]$ satisfies the $m$ th cancellation condition $C_{m}$ if for no $m$ distinct comparisons $A_{i} \succeq B_{i}, i=1,2, \ldots, m$, among which $A_{i} \succ B_{i}$ for at least one $i$, there exist positive integers $a_{1}, \ldots, a_{m}$ such that the following two multiset unions coincide

$$
\bigcup_{i=1}^{m} \underbrace{\left(A_{i} \cup \ldots \cup A_{i}\right)}_{a_{i}}=\bigcup_{i=1}^{m} \underbrace{\left(B_{i} \cup \ldots \cup B_{i}\right)}_{a_{i}} .
$$

This is a complete analogue of the $m$ th cancellation condition formulated by Kraft, Pratt and Seidenberg [?] for orders on the power set $2^{[n]}$. As in [?, Theorem 2] it is easy to show that for an order $\succeq$ on $\mathcal{P}_{k}[n]$ to be representable it is necessary and sufficient that all cancellation conditions $C_{2}, C_{3}, \ldots$ are satisfied. This will become obvious later, when we reformulate this statement in vector form, in which the cancellation conditions look much more natural.

Example 2. The nonrepresentable linear order $A_{4}$ on $\mathcal{P}_{2}[4]$ given by

$$
\left\{1^{2}\right\} \succ\{12\} \succ \underline{\{13\} \succ\left\{2^{2}\right\}} \succ \underline{\{23\} \succ\{14\}} \succ \underline{\{24\} \succ\left\{3^{2}\right\}} \succ\{34\} \succ\left\{4^{2}\right\}
$$


and constructed in [?] does not satisfy the condition $C_{3}$, since it contains the following comparisons (underlined above):

$$
\{13\} \succ\left\{2^{2}\right\}, \quad\{23\} \succ\{14\}, \quad\{24\} \succ\left\{3^{2}\right\} .
$$

Indeed, the union of the multisets on the right and the union of the multisets on the left are both equal to the multiset $\left\{12^{2} 3^{2} 4\right\}$. Thus $C_{3}$ is violated with $a_{1}=a_{2}=a_{3}=1$.

In order to convince the reader that the above cancellation conditions are natural, we note that the IES for $\succeq$ follows from $C_{2}$, since it cannot be true that $A \cup W_{1} \succeq B \cup W_{1}$ and $B \cup W_{2} \succ A \cup W_{2}$. When $W_{1}=W_{2}=\emptyset$ we obtain also antisymmetry. It is also obvious that $C_{2}$ follows from the IES and antisymmetry, and hence for antisymmetric orders, condition $C_{2}$ and the IES are equivalent. Transitivity of $\succeq$ is implied by $C_{3}$ as it is impossible to have simultaneously $A \succeq B, B \succeq C$, and $C \succ A$.

It should be noted that, although transitivity follows from $C_{3}$, it is weaker than $C_{3}$ (see Example ??). It is of special interest when the IES implies $C_{3}, C_{4}, \ldots$, and hence representability of $\succeq$. For $n=3$, Sertel and Slinko [?] showed that the IES implies $C_{i}$ for all $i \geq 3$, and hence all orders on $\mathcal{P}_{k}[3]$ which satisfy the IES are representable, for all $k \geq 1$. This is no longer true for $n>3$ as shown in the same paper. Nevertheless, sometimes something good can be said about $\succeq$ even when it is not representable.

Definition 5. We will say that an order $\succeq$ on $\mathcal{P}_{k}[n]$ is almost representable if there exist nonnegative real numbers $u_{1}, \ldots, u_{m}$ such that for all $M_{1}=$ $\left([n], \mu_{1}\right)$ and $M_{2}=\left([n], \mu_{2}\right)$ belonging to $\mathcal{P}_{k}[n]$,

$$
M_{1} \succeq M_{2} \Longrightarrow \sum_{i=1}^{n} \mu_{1}(i) u_{i} \geq \sum_{i=1}^{n} \mu_{2}(i) u_{i}
$$

This is a complete analogue of the almost representability condition for comparative probability orders [?, ?].

Almost representable orders satisfying the IES need not be representable (see [?, Sect. 3]). Such linear orders may exist only when the representable order associated with utilities $u_{1}, \ldots, u_{m}$ is non-linear and some multisets are tied. There are also linear orders which satisfy the IES but still fail to be almost representable (see [?, Sect. 4]). More precisely, examples are given in [?] of a non-representable but almost representable linear order in $\mathcal{P}_{2}$ [4], and a linear order in $\mathcal{P}_{3}[4]$ that fails to be almost representable; both satisfy the IES but fail to satisfy $C_{3}$. 
Thus it is clear that to secure representability, sometimes we have to assume more than the IES (or $C_{2}$, which is the same), and we may need also some of the conditions $C_{3}, C_{4}, \ldots$ It is important to find out how far do we have to go, and how many cancellation conditions we need to assume before we can guarantee the representability of an order on $\mathcal{P}_{k}[n]$. The current paper is devoted to this particular question. All results will be formulated in terms of the following two functions.

For $n \geq 3$ and $k \geq 2$, let $f(n, k)$ be the smallest positive integer such that any linear order on $\mathcal{P}_{k}[n]$ which satisfies $C_{2}, C_{3}, \ldots, C_{f(n, k)}$ is representable. Similarly, let $g(n, k)$ be the smallest positive integer such that any almost representable linear order on $\mathcal{P}_{k}[n]$ which satisfies $C_{2}, C_{3}, \ldots, C_{g(n, k)}$ is representable. Clearly, $g(n, k) \leq f(n, k)$ for all $n$ and $k$. Sertel and Slinko [?] showed in this notation that $f(3, k)=g(3, k)=2$ for all $k \geq 1$.

In this paper we will completely describe the function $g(n, k)$. Specifically, we will show that $g(n, k)=n-1$ for all $n \geq 3$ and $k \geq 2$ apart from the pair $(n, k)=(5,2)$, for which $g(5,2)=3$. As for the function $f(n, k)$, the best we can prove in general is that $n-1 \leq f(n, k) \leq n$ whenever $(n, k) \neq(5,2)$. Computer-assisted calculations show that $g(n, k)=f(n, k)$ for small values of $n$ and $k$ (namely, for $(n, k)=(4,2),(4,3),(5,2),(5,3),(6,2)$ and $(7,2)$ ), and so we conjecture that this is true in general.

To obtain these results we take a geometric view of linear orders, and define discrete cones (similar to those defined by Fishburn [?] for comparative probability orders on a set). We will show that to every order $\succeq$ on $\mathcal{P}_{k}[n]$ satisfying the IES there corresponds a discrete cone $C(\succeq)$, and vice versa.

We are grateful to an anonymous referee and the action editor Jean-Paul Doignon for numerous valuable suggestions.

\section{Discrete Cones}

To define discrete cones we need the following notation. Let $\left\{\mathbf{e}_{1}, \ldots, \mathbf{e}_{n}\right\}$ be the standard basis of $\mathbb{R}^{n}$, let $M_{k}=\{-k, \ldots,-1,0,1, \ldots, k\}$ and let $M_{k}^{n}$ be the $n$th Cartesian product of $M_{k}$. Next, for $\mathbf{x}=\left(x_{1}, \ldots, x_{n}\right) \in M_{k}^{n}$, define

$$
\begin{aligned}
|\mathbf{x}| & =\sum_{i=1}^{n}\left|x_{i}\right|, \\
\mathbf{x}^{+} & =\left(v_{1}, \ldots, v_{n}\right), \quad \text { where } v_{i}=\max \left(x_{i}, 0\right), \text { and } \\
\mathbf{x}^{-} & =\left(u_{1}, \ldots, u_{n}\right), \quad \text { where } u_{i}=\min \left(x_{i}, 0\right),
\end{aligned}
$$


so that $\mathbf{x}=\mathbf{x}^{+}+\mathbf{x}^{-}$and $|\mathbf{x}|=\left|\mathbf{x}^{+}\right|+\left|\mathbf{x}^{-}\right|$. Similarly, for any two vectors $\mathbf{x}=\left(x_{1}, \ldots, x_{n}\right) \in M_{k}^{n}$ and $\mathbf{y}=\left(y_{1}, \ldots, y_{n}\right) \in M_{k}^{n}$, let

$$
\max (\mathbf{x}, \mathbf{y})=\left(w_{1}, \ldots, w_{n}\right), \quad \text { where } w_{i}=\max \left(x_{i}, y_{i}\right) .
$$

Finally, we define $T_{k}^{n}$, a set in which the cones associated with orders on $\mathcal{P}_{k}[n]$ will live, by

$$
T_{k}^{n}=\left\{\mathbf{x} \in M_{k}^{n}|| \mathbf{x}^{-}|=| \mathbf{x}^{+} \mid \leq k\right\} .
$$

This consists of all vectors in $M_{k}^{n}$ which are orthogonal to the vector $\mathbf{n}=$ $(1,1, \ldots, 1)$ and whose sum of positive entries is no greater than $k$.

Given a multiset $M=([n], \mu) \in \mathcal{P}_{k}[n]$, let $\chi(M)=(\mu(1), \ldots, \mu(n))$ be the characteristic function of $M$. Given an order $\succeq$ on $\mathcal{P}_{k}[n]$, for every comparison $M \succeq N$ we construct a vector $\chi(M, N)=\chi(M)-\chi(N) \in T_{k}^{n}$. We can now define $C(\succeq)$ to be the set of all vectors $\chi(M, N)$ for all valid comparisons $M \succeq N$, where $M, N \in \mathcal{P}_{k}[n]$. Note that, if $M \cap N \neq \emptyset$, then for $M^{\prime}=M \backslash(M \cap N)$ and $N^{\prime}=N \backslash(M \cap N)$ we have $\chi(M, N)=\chi\left(M^{\prime}, N^{\prime}\right)$. This means that for any order $\succeq$ which satisfies the IES, then $C(\succeq)$ is welldefined. Now we will give an abstract definition of this object.

Definition 6. A subset $C \subseteq T_{k}^{n}$ is said to be a discrete cone (or simply, a cone) in $T_{k}^{n}$ if the following conditions hold:

D1. $\mathbf{e}_{i}-\mathbf{e}_{i+1} \in C \backslash(-C)$ for $1 \leq i \leq n-1$,

D2. $\mathbf{0} \in C$, and for all $\mathbf{x} \in T_{k}^{n} \backslash\{\mathbf{0}\}$ either $\mathbf{x} \in C$ or $-\mathbf{x} \in C$ (but not both),

D3. $\mathbf{x}+\mathbf{y} \in C$ whenever $\mathbf{x}, \mathbf{y} \in C$ and $\left|\max \left(-\mathbf{x}^{-}, \mathbf{y}^{+}\right)\right| \leq k$.

We note that if $m \leq k$ and $\succeq_{m}$ is the order on $\mathcal{P}_{m}[n]$ associated with $\succeq$, then $C\left(\succeq_{m}\right) \subseteq C(\succeq)$. Furthermore:

Proposition 1. If a linear order $\succeq$ on $\mathcal{P}_{k}[n]$ satisfies (??) and the IES, then $C(\succeq)$ satisfies conditions D1 to D3 and so is a discrete cone. On the other hand, every discrete cone $C \subseteq T_{k}^{n}$ defines a linear order $\succeq$ on $\mathcal{P}_{k}[n]$ which satisfies (??) and the IES, and in which $A \succeq B$ if and only if $\chi(A, B) \in C$, for every two multisets $A, B \in \mathcal{P}_{k}[n]$.

Proof. Let $\succeq$ be a linear order on $\mathcal{P}_{k}[n]$ which satisfies (??) and the IES. Then (??) implies conditions D1 for $C(\succeq)$ and D2 obviously holds for $C(\succeq)$ since $\succeq$ is linear. Only D3 is not immediately clear.

So suppose that $\mathbf{x}=\left(x_{1}, \ldots, x_{n}\right)=\chi(A, B) \in C(\succeq)$ and that $\mathbf{y}=$ $\left(y_{1}, \ldots, y_{n}\right)=\chi(D, E) \in C(\succeq)$, with $\left|\max \left(-\mathbf{x}^{-}, \mathbf{y}^{+}\right)\right| \leq k$. We may choose 
such representations of $\mathbf{x}$ and $\mathbf{y}$ for which $A \cap B=\emptyset$ and $D \cap E=\emptyset$. In doing this, suppose that $A$ and $B$ have cardinality $s$, and $D$ and $E$ have cardinality $t$, where $s \leq k$ and $t \leq k$. Then $\mathbf{x}^{+}=\chi(A)$ and $-\mathbf{x}^{-}=\chi(B)$ while $\mathbf{y}^{+}=\chi(D)$ and $-\mathbf{y}^{-}=\chi(E)$, and $\left|\mathbf{x}^{-}\right|=\left|\mathbf{x}^{+}\right|=|A|=|B|=s$ while $\left|\mathbf{y}^{-}\right|=\left|\mathbf{y}^{+}\right|=|D|=|E|=t$.

This means that $A, B \in \mathcal{P}_{s}[n]$ and $D, E \in \mathcal{P}_{t}[n]$ with $A \succeq_{s} B$ and $D \succeq_{t} E$, where $\succeq_{s}$ and $\succeq_{t}$ are the orders induced by $\succeq$ on $\mathcal{P}_{s}[n]$ and $\mathcal{P}_{t}[n]$, respectively. The fact that $\left|\max \left(-\mathbf{x}^{-}, \mathbf{y}^{+}\right)\right| \leq k$ implies that there exist two multisets $F_{1}$ and $F_{2}$ such that $F_{1} \cup B=F_{2} \cup D$, and the cardinality $\ell$ of this common union is not greater than $k$. By Lemma ??,

$$
F_{1} \cup A \succeq_{\ell} F_{1} \cup B=F_{2} \cup D \succeq_{\ell} F_{2} \cup E,
$$

and hence by transitivity, $F_{1} \cup A \succeq_{\ell} F_{2} \cup E$. It now follows that

$$
\begin{aligned}
\mathbf{x}+\mathbf{y} & =\chi(A \cup D, B \cup E)=\chi\left(A \cup F_{2} \cup D, B \cup F_{2} \cup E\right) \\
& =\chi\left(A \cup F_{1} \cup B, B \cup F_{2} \cup E\right)=\chi\left(A \cup F_{1}, F_{2} \cup E\right) \in C(\succeq) .
\end{aligned}
$$

For the second part, let $C$ be any cone in $T_{k}^{n}$. We define an order on each of the sets $\mathcal{P}_{\ell}[n]$ for $\ell \leq k$ by setting for any two multisets $A, B \in \mathcal{P}_{\ell}[n]$

$$
A \succeq_{\ell} B \text { whenever } \chi(A, B) \in C .
$$

By its definition, the order $\succeq_{k}(=\succeq)$ on $\mathcal{P}_{k}[n]$ will satisfy the IES, but we need to prove its transitivity. So suppose $A \succeq B$ and $B \succeq D$ for some $A, B, D \in$ $\mathcal{P}_{k}[n]$. Let $\mathbf{x}=\chi(A, B)$ and $\mathbf{y}=\chi(B, D)$. Then $\left|\max \left(-\mathbf{x}^{-}, \mathbf{y}^{+}\right)\right| \leq k$ since each entry of both $-\mathbf{x}^{-}$and $\mathbf{y}^{+}$is bounded above by the corresponding entry of $\chi(B)$, and $|\chi(B)|=k$, so $\chi(A, D)=\chi(A)-\chi(B)+\chi(B)-\chi(D)=\mathbf{x}+\mathbf{y} \in$ $C$, and therefore $A \succeq D$.

Example 3. For the linear order $\succeq$ on $\mathcal{P}_{2}[4]$ from Example ??, the three vectors

$$
\mathbf{x}=(1,-2,1,0), \mathbf{y}=(0,1,-2,1), \mathbf{z}=(-1,1,1,-1)
$$

belong to $C(\succeq)$, which is a cone in $T_{2}^{4}$. These vectors correspond to the three comparisons given in (??). Note that for each pair of distinct vectors $\mathbf{u}, \mathbf{v}$ in the set $\{\mathbf{x}, \mathbf{y}, \mathbf{z}\}$ we have $\left|\max \left(\mathbf{u}^{+}, \mathbf{v}^{-}\right)\right|=3$, and that explains why no sum of two vectors from this set belongs to $C(\succeq)$.

We can reformulate now the cancellation conditions as follows:

Proposition 2. An order $\succeq$ satisfies the mth cancellation condition if and only if for no m-subset $\left\{\mathbf{x}_{1}, \ldots, \mathbf{x}_{m}\right\} \subseteq C(\succeq)$ do there exist positive integers $a_{1}, \ldots, a_{m}$ such that

$$
a_{1} \mathbf{x}_{1}+a_{2} \mathbf{x}_{2}+\cdots+a_{m} \mathbf{x}_{m}=0
$$


Proof. If $m$ distinct comparisons $A_{i} \succeq B_{i}$ exist as in Definition ??, then we take vectors $\mathbf{x}_{i}=\chi\left(A_{i}, B_{i}\right) \in C(\succeq)$ for $1 \leq i \leq m$, and condition (??) implies that $a_{1} \mathbf{x}_{1}+a_{2} \mathbf{x}_{2}+\cdots+a_{m} \mathbf{x}_{m}=\mathbf{0}$. The converse is also clear.

Definition 7. Suppose for an order $\succeq$ on $\mathcal{P}_{k}[n]$ there exists a set of $m$ vectors $\left\{\mathbf{x}_{1}, \ldots, \mathbf{x}_{m}\right\} \subseteq C(\succeq)$ and positive integers $a_{1}, \ldots, a_{m}$ such that (??) holds. Then we say that the order $\succeq$ violates the mth cancellation condition for vectors $\mathbf{x}_{1}, \ldots, \mathbf{x}_{m}$ with multiplicities $\left(a_{1}, \ldots, a_{m}\right)$.

Example 4. The three vectors from $C(\succeq)$ given in Example ?? add up to the zero vector, hence the order $\succeq$ violates $C_{3}$ with multiplicities $(1,1,1)$, and is not representable.

Geometrically, what is happening is clear. An order $\succeq$ is representable provided there exists a positive-integer-valued vector $\mathbf{w}=\left(w_{1}, \ldots, w_{n}\right) \in \mathbb{R}^{n}$ such that $w_{1}>w_{2}>\ldots>w_{n}$ and such that

$$
\mathbf{x} \in C(\succeq) \Longleftrightarrow(\mathbf{w}, \mathbf{x})>0 \text { for every } \mathbf{x} \in T_{k}^{n} \backslash\{\mathbf{0}\},
$$

that is, all non-zero vectors in the cone $C(\succeq)$ lie in the open half-space $H_{\mathbf{w}}=\left\{\mathbf{x} \in \mathbb{R}^{n} \mid(\mathbf{w}, \mathbf{x})>0\right\}$ determined by the hyperplane $L_{\mathbf{w}}$ with normal vector $\mathbf{w}$. The cone $C(\succeq)$ in this case is a pointed cone.

Similarly, for any order $\succeq$ that is almost representable, there exists a nonnegative-integer-valued vector $\mathbf{w}=\left(w_{1}, \ldots, w_{n}\right) \in \mathbb{R}^{n}$ such that both $w_{1} \geq w_{2} \geq \ldots \geq w_{n} \geq 0$ and

$$
\mathbf{x} \in C(\succeq) \Longrightarrow(\mathbf{w}, \mathbf{x}) \geq 0 \text { for every } \mathbf{x} \in T_{k}^{n}
$$

so that in this case the whole of the cone $C(\succeq)$ lies in the closed half-space $\overline{H_{\mathbf{w}}}=\left\{\mathbf{x} \in \mathbb{R}^{n} \mid(\mathbf{w}, \mathbf{x}) \geq 0\right\}=H_{\mathbf{w}} \cup L_{\mathbf{w}}$.

Note that in both cases, the vector $\mathbf{w}$ represents the vector of utilities.

\section{Construction Theorem}

Proposition 3. Let $\succeq$ be an almost representable order with a vector of utilities $\mathbf{w}$. Suppose that the $m$ th cancellation condition is violated for vectors $\mathbf{x}_{1}, \ldots, \mathbf{x}_{m}$ with multiplicities $\left(a_{1}, \ldots, a_{m}\right)$. Then all the vectors $\mathbf{x}_{1}, \ldots, \mathbf{x}_{m}$ lie in the hyperplane $L_{\mathbf{w}}$.

Proof. First we note that $(\mathbf{w}, \mathbf{x})>0$ for all those $\mathbf{x} \in C(\succeq)$ which do not belong to $L_{\mathbf{w}}$. Next, condition (??) implies that

$$
\sum_{i=1}^{n} a_{i}\left(\mathbf{w}, \mathbf{x}_{i}\right)=0
$$


and since $a_{i}>0$ for $1 \leq i \leq n$, this can hold only when all $\left(\mathbf{w}, \mathbf{x}_{i}\right)=0$ for all $i$, and hence we find that $\mathbf{x}_{i} \in L_{\mathbf{w}}$ for $1 \leq i \leq n$.

Corollary 1. Any almost representable order on $\mathcal{P}_{k}[n]$ which satisfies $C_{3}$, $C_{4}, \ldots, C_{n-1}$ is representable.

Proof. Suppose that for some $m$, the $m$ th cancellation condition is violated by vectors $\mathbf{x}_{1}, \ldots, \mathbf{x}_{m} \in C(\succeq)$ with multiplicities $\left(a_{1}, \ldots, a_{m}\right)$, that is, with $a_{1} \mathbf{x}_{1}+\cdots+a_{m} \mathbf{x}_{m}=\mathbf{0}$. Now since all the vectors $\mathbf{x}_{1}, \ldots, \mathbf{x}_{m}$ belong to the $(n-2)$-dimensional subspace $L_{\mathbf{w}} \cap L_{\mathbf{n}}$, where $L_{\mathbf{n}}$ is the hyperplane with normal vector $\mathbf{n}=(1,1, \ldots, 1)$, we may use standard linear algebra to reduce the number of vectors in this linear combination to at most $n-1$ vectors, while keeping all coefficients positive. We claim that if $m \geq n$, then one of the vectors $\mathbf{x}_{1}, \ldots, \mathbf{x}_{m}$ can be excluded from the linear combination. Indeed in this case the set of vectors $\left\{\mathbf{x}_{1}, \ldots, \mathbf{x}_{m-1}\right\}$ is linearly dependent, and so there exist real numbers $b_{1}, \ldots, b_{m-1}$ with at least one $b_{i}$ being positive, such that $b_{1} \mathbf{x}_{1}+\cdots+b_{m-1} \mathbf{x}_{m-1}=\mathbf{0}$. Hence for all $\epsilon \in \mathbb{R}$ we have

$$
\left(a_{1}-\epsilon b_{1}\right) \mathbf{x}_{1}+\ldots+\left(a_{m}-\epsilon b_{m}\right) \mathbf{x}_{m}+a_{m} \mathbf{x}_{m}=\mathbf{0} .
$$

When $\epsilon=0$, all coefficients in this equation are positive. If we choose the smallest positive $\epsilon$ for which $a_{i}-\epsilon b_{i}=0$ for some $i$, then the resulting linear combination will not contain $\mathbf{x}_{i}$, and will still be nontrivial since $a_{m}>0$. Hence we may suppose that $m \leq n-1$. Since $\succeq$ satisfies $C_{4}, \ldots, C_{n-1}$, it follows that $\succeq$ is representable.

Proposition 4. $g(n, k) \leq n-1$ and $f(n, k) \leq n$ for all $n$ and $k$.

Proof. The first inequality $g(n, k) \leq n-1$ follows from Corollary ??, and the second inequality $f(n, k) \leq n$ follows from a similar argument in the $(n-1)$-dimensional subspace $L_{\mathbf{n}}$.

The following theorem enables us to construct orderings that are almost representable but fail $C_{m}$ for particular values of $m$.

Theorem 1 (Construction method). For $m \geq 3$, let $X=\left\{\mathbf{x}_{1}, \ldots, \mathbf{x}_{m}\right\}$ be a system of $m$ non-zero vectors from $T_{k}^{n}$ satisfying the following conditions:

(a) $\sum_{i=1}^{m} a_{i} \mathbf{x}_{i}=\mathbf{0}$ for some positive integers $a_{1}, \ldots, a_{m}$,

(b) $\left|\max \left(-\mathbf{x}_{i}^{-}, \mathbf{x}_{j}^{+}\right)\right|>k$ for every pair $\{i, j\} \subset\{1,2, \ldots, m\}$ with $i \neq j$, and 
(c) no proper subsystem $X^{\prime} \subset X$ is linearly dependent with positive coefficients.

Suppose further that the $m \times n$ matrix $A$ whose rows are the given vectors $\mathbf{x}_{1}, \ldots, \mathbf{x}_{m}$ has the property that $A \mathbf{w}=\mathbf{0}$ for some positive-integer-valued vector $\mathbf{w}=\left(w_{1}, \ldots, w_{n}\right)$ with $w_{1}>w_{2}>\ldots>w_{n}>0$, and that

$$
L_{\mathbf{w}} \cap\left(T_{k}^{n} \backslash\{\mathbf{0}\}\right)=\left\{ \pm \mathbf{x}_{1}, \ldots, \pm \mathbf{x}_{m}\right\} .
$$

Let $\succeq$ be the (nonlinear) order on $\mathcal{P}_{k}[n]$ with the vector of utilities $\mathbf{w}$, and let $C(\succeq)=\left\{\mathbf{x} \in T_{k}^{n} \mid(\mathbf{x}, \mathbf{w}) \geq 0\right\}$ be the discrete cone associated with $\succeq$. Then the set

$$
C^{\prime}=C(\succeq) \backslash\left\{-\mathbf{x}_{1}, \ldots,-\mathbf{x}_{m}\right\}
$$

is a discrete cone that corresponds to an almost representable order on $\mathcal{P}_{k}[n]$ which satisfies $C_{j}$ for all $j<m$, but does not satisfy $C_{m}$.

Proof. First we note that $\left\{ \pm \mathbf{x}_{1}, \ldots, \pm \mathbf{x}_{m}\right\} \subseteq L_{\mathbf{w}}=\left\{\mathbf{x} \in \mathbb{R}^{n} \mid(\mathbf{w}, \mathbf{x})=0\right\}$. Next $\left(\mathbf{e}_{i}-\mathbf{e}_{i+1}, \mathbf{w}\right)=w_{i}-w_{i+1}>0$ for $1 \leq i<n$, so the property D1 holds for the cone $C^{\prime}$. Property D2 holds for $C^{\prime}$ because it holds for $C(\succeq)$ and, when we remove vectors $-\mathbf{x}_{1}, \ldots,-\mathbf{x}_{m}$ from $C^{\prime}$, the vectors $\mathbf{x}_{1}, \ldots, \mathbf{x}_{m}$ remain in $C^{\prime}$. Now let us prove D3. Suppose that $\mathbf{y}, \mathbf{z} \in C^{\prime}$ and $\left|\max \left(-\mathbf{y}^{-}, \mathbf{z}^{+}\right)\right| \leq k$, but $\mathbf{y}+\mathbf{z} \notin C^{\prime}$. Since the cone $C(\succeq)$ satisfies D3 we know that $\mathbf{y}+\mathbf{z} \in \bar{C}(\succeq)$, so $\mathbf{y}+\mathbf{z} \in C(\succeq) \backslash C^{\prime}$, and hence $\mathbf{y}+\mathbf{z}=-\mathbf{x}_{i}$ for some $i$. Moreover, $\mathbf{y}, \mathbf{z} \in L_{\mathbf{w}}$, since otherwise $-\left(\mathbf{x}_{i}, \mathbf{w}\right)=(\mathbf{y}+\mathbf{z}, \mathbf{w})=(\mathbf{y}, \mathbf{w})+(\mathbf{z}, \mathbf{w})>0$. It follows from (c) that $\mathbf{y}=\mathbf{x}_{j}$ for some $j$ and $\mathbf{z}=\mathbf{x}_{\ell}$ for some $\ell$. This contradicts hypothesis (b), however, since $\left|\max \left(-\mathbf{y}^{-}, \mathbf{z}^{+}\right)\right| \leq k$. Thus D3 holds for $C^{\prime}$.

Suppose now that some violation of $C_{j}$ occurs, say

$$
c_{1} \mathbf{y}_{1}+c_{2} \mathbf{y}_{2}+\cdots+c_{j} \mathbf{y}_{j}=\mathbf{0}
$$

with positive integers $c_{1}, \ldots, c_{j}$ and $\mathbf{y}_{i} \in C^{\prime}$ for $1 \leq i \leq j$, where $j<m$. If $\left(\mathbf{y}_{i}, \mathbf{w}\right)>0$ for some $i$, then $0=(\mathbf{0}, \mathbf{w})=\left(c_{1} \mathbf{y}_{1}+c_{2} \mathbf{y}_{2}+\cdots+c_{j} \mathbf{y}_{j}, \mathbf{w}\right)=$ $c_{1}\left(\mathbf{y}_{1}, \mathbf{w}\right)+c_{2}\left(\mathbf{y}_{2}, \mathbf{w}\right)+\ldots+c_{j}\left(\mathbf{y}_{j}, \mathbf{w}\right)>0$, a contradiction, hence $\mathbf{y}_{i} \in L_{\mathbf{w}}$ for all $i$, and therefore $\left\{\mathbf{y}_{1}, \ldots, \mathbf{y}_{j}\right\} \subseteq\left\{\mathbf{x}_{1}, \ldots, \mathbf{x}_{m}\right\}$, which contradicts (c). Finally, $C_{m}$ fails by hypothesis (a).

\section{Characterisation of $g(n, k)$}

In this section we will use the construction method from the previous section and Proposition ?? to characterise the function $g(n, k)$ as follows: 
Theorem 2. For all $n \geq 3$ and $k \geq 2$,

$$
g(n, k)= \begin{cases}n-2 & \text { if }(n, k)=(5,2) \\ n-1 & \text { otherwise }\end{cases}
$$

We prove this theorem partly by theoretical means and partly by direct calculations for small $n$ and $k$ (with the help of the computer algebra system MAGMA [?], using the same techniques of enumeration as described in [?]). We present the theoretical results first.

As was mentioned above, the case $n=3$ of this theorem was proved in [?], so we need only consider cases where $n \geq 4$. The proof of each case will consist of constructing a matrix $A$ which satisfy conditions of Theorem 1 . The rows of $A$ will be denoted by $\mathbf{v}_{1}, \mathbf{v}_{2}, \ldots$. We will use the following notation extensively: for any vector $\mathbf{v}$, let the expression $x_{i}(\mathbf{v})$ denote the $i$ th co-ordinate entry of $\mathbf{v}$.

\subsection{Proof of Theorem ?? for $n=4$.}

Consider the vectors $\mathbf{v}_{1}, \mathbf{v}_{2}, \mathbf{v}_{3}$ that make up the rows of the $3 \times 4$ matrix

$$
A=\left[\begin{array}{rccc}
0 & -(k-1) & k & -1 \\
1 & -1 & -(k-1) & k-1 \\
-1 & k & -1 & -k+2
\end{array}\right]
$$

It is clear that $\mathbf{v}_{1}$ and $\mathbf{v}_{2}$ are linearly independent and $\mathbf{v}_{1}+\mathbf{v}_{2}+\mathbf{v}_{3}=\mathbf{0}$. It can also be seen (by inspection of the first and fourth co-ordinates) that no linear combination $y_{1} \mathbf{v}_{1}+y_{2} \mathbf{v}_{2}$ of $\mathbf{v}_{1}$ and $\mathbf{v}_{2}$ can be a vector of integers unless both $y_{1}$ and $y_{2}$ are integers. The row-space of $A$ has a normal vector $\mathbf{w}=$ $\left(k^{2}-k+2, k+1, k, 1\right)$, satisfying the requirement $w_{1}>w_{2}>\ldots>w_{n}>0$ of Theorem ?? since $k^{2}-k+1>k$ for all $k>1$. Similarly the given vectors $\mathbf{v}_{1}, \mathbf{v}_{2}, \mathbf{v}_{3}$ satisfy the requirement that $\left|\max \left(-\mathbf{v}_{i}^{-}, \mathbf{v}_{j}^{+}\right)\right|>k$ for every two $i$ and $j$, since $2 k-1>k$ for all $k>1$.

Next, because $\left|\mathbf{v}_{1}^{+}\right|=\left|\mathbf{v}_{1}^{-}\right|=\left|\mathbf{v}_{2}^{+}\right|=\left|\mathbf{v}_{2}^{-}\right|=k$, no proper multiple of $\mathbf{v}_{1}$ or $\mathbf{v}_{2}$ can lie in $T_{k}^{4}$, and hence any vector $\mathbf{v}$ in $L_{\mathbf{w}} \cap T_{k}^{4}$ other than $\mathbf{0}, \pm \mathbf{v}_{1}$ or $\pm \mathbf{v}_{2}$ must be of the form $\mathbf{v}=m_{1} \mathbf{v}_{1}+m_{2} \mathbf{v}_{2}$ with non-zero coefficients $m_{1}, m_{2} \in \mathbb{Z}$. Now if $m_{1}>0$ and $m_{2}>0$, then since $-k \leq x_{2}(\mathbf{v})=-m_{1}(k-1)-m_{2}<0$ we find that $m_{1}=m_{2}=1$, giving $\mathbf{v}=(1,-k, 1, k-2)=-\mathbf{v}_{3}$. Similarly, if $m_{1}<0$ and $m_{2}<0$, then inspection of $x_{2}(\mathbf{v})$ gives $m_{1}=m_{2}=-1$ and $\mathbf{v}=\mathbf{v}_{3}$. On the other hand, if $m_{1}>0>m_{2}$ or $m_{1}<0<m_{2}$ then $x_{3}(\mathbf{v})=m_{1} k-m_{2}(k-1)$ lies outside the acceptable range $M_{k}$ for co-ordinates of vectors in $T_{k}^{4}$. Hence the only non-zero vectors in $L_{\mathbf{w}} \cap T_{k}^{4}$ are $\pm \mathbf{v}_{1}, \pm \mathbf{v}_{2}$ and $\pm \mathbf{v}_{3}$. 
By Theorem ??, these vectors give rise to an almost representable order on $\mathcal{P}_{k}[4]$ which fails to satisfy $C_{3}$, and by Proposition ?? it follows that $g(4, k)=3$ for all $k \geq 2$.

\subsection{Proof of Theorem ?? for $n=5$ and $k \geq 4$.}

Consider the four vectors $\mathbf{v}_{1}, \mathbf{v}_{2}, \mathbf{v}_{3}, \mathbf{v}_{4}$ forming the rows of the $4 \times 5$ matrix

$$
A=\left[\begin{array}{ccccc}
1 & -(k-1) & k-3 & 2 & -1 \\
0 & 1 & -k & k-2 & 1 \\
0 & 0 & 1 & -k & k-1 \\
-1 & k-2 & 2 & 0 & -(k-1)
\end{array}\right]
$$

We note that $\mathbf{v}_{1}+\mathbf{v}_{2}+\mathbf{v}_{3}+\mathbf{v}_{4}=\mathbf{0}$, any three of the given vectors are linearly independent, and the vector $\mathbf{w}=\left(k^{3}-3 k^{2}+6 k-3, k^{2}-k+3, k+1,2,1\right)$ is a normal vector to the row-space of $A$. Also the given vectors can easily be seen to satisfy the requirement that $\left|\max \left(-\mathbf{v}_{i}^{-}, \mathbf{v}_{j}^{+}\right)\right|>k$ whenever $i \neq j$. Next, we need the following:

Lemma 2. $L_{\mathbf{w}} \cap T_{k}^{5}=\left\{\mathbf{0}, \pm \mathbf{v}_{1}, \pm \mathbf{v}_{2}, \pm \mathbf{v}_{3}, \pm \mathbf{v}_{4}\right\}$.

Proof. Clearly $\left\{\mathbf{v}_{1}, \mathbf{v}_{2}, \mathbf{v}_{3}\right\}$ is a basis for the 3-dimensional subspace $L_{\mathbf{w}} \cap L_{\mathbf{n}}$, so every vector $\mathbf{v}$ in $L_{\mathbf{w}} \cap T_{k}^{5}$ must be of the form $\mathbf{v}=y_{1} \mathbf{v}_{1}+y_{2} \mathbf{v}_{2}+y_{3} \mathbf{v}_{3}$, and moreover, from inspection of $x_{1}(\mathbf{v}), x_{2}(\mathbf{v})$ and $x_{3}(\mathbf{v})$ it is obvious that $y_{1}, y_{2}$ and $y_{3}$ have to be integers. Without loss of generality ( since $-T_{k}^{5}=T_{k}^{5}$ ), we may assume that $y_{1} \geq 0$. We proceed case-by-case:

Case 1: Suppose $y_{1}=0$. If $y_{2} \neq 0$ then $y_{2}$ and $y_{3}$ must have the same sign (for otherwise $x_{3}(\mathbf{v})=-y_{2} k+y_{3}$ lies outside $M_{k}$ ), and then inspection of $x_{2}(\mathbf{v})+x_{5}(\mathbf{v})=2 y_{2}+(k-1) y_{3}$ forces $y_{3}=0$ (because membership of $T_{k}^{5}$ requires $\left.\left|\mathbf{v}^{-}\right|=\left|\mathbf{v}^{+}\right| \leq k\right)$. Hence either $y_{2}=0$ or $y_{3}=0$, giving $\mathbf{v}=\mathbf{0}, \pm \mathbf{v}_{2}$ or $\pm \mathbf{v}_{3}$.

Case 2: Suppose $y_{1}>0$ and $y_{2}=0$. Then inspection of $x_{2}(\mathbf{v})=-y_{1}(k-1)$ gives $y_{1}=1$. Also if $y_{3} \neq 0$ then $y_{3}>0$ (for otherwise $x_{4}(\mathbf{v})=2 y_{1}-y_{3} k>k$ ), but then $\mathbf{v}^{-}=\left(0,-(k-1), 0,2-y_{3} k, 0\right)$ so $\left|\mathbf{v}^{-}\right|=k-3+y_{3} k>k$, a contradiction. Hence $\mathbf{v}=\mathbf{v}_{1}$ in this case.

Case 3: Suppose $y_{1}>0, y_{2} \neq 0$ and $y_{3}=0$. Then inspection of $x_{3}(\mathbf{v})=$ $y_{1}(k-3)-y_{2} k$ gives $y_{2}>0$, following which inspection of $x_{4}(\mathbf{v})=2 y_{1}+$ $y_{2}(k-2)$ gives $y_{1}=y_{2}=1$, but then $\mathbf{v}=\mathbf{v}_{1}+\mathbf{v}_{2}=(1,-(k-2),-3, k, 0)$ so $\left|\mathbf{v}^{+}\right|=1+k>k$, a contradiction. Hence no such $\mathbf{v}$ exists in this case.

Case 4: Suppose $y_{1}>0, y_{2}<0$ and $y_{3} \neq 0$. Then since $-k \leq x_{2}(\mathbf{v})=$ $-y_{1}(k-1)+y_{2}<0$ we find that $y_{1}=1$ and $y_{2}=-1$, and therefore 
$\mathbf{v}=\left(1,-k, 2 k-3+y_{3}, 4-k\left(1+y_{3}\right),-2+y_{3}(k-1)\right)$. Now as $x_{2}(\mathbf{v})=-k$, no other co-ordinates of $\mathbf{v}$ can be negative, so $4-k\left(1+y_{3}\right)=x_{4}(\mathbf{v}) \geq 0$ and $-2+y_{3}(k-1)=x_{5}(\mathbf{v}) \geq 0$. The first of these two inequalities gives $y_{3} \leq 0$ but the second gives $y_{3}>0$, a contradiction, so no such $\mathbf{v}$ exists in this case.

Case 5: Suppose $y_{1}>0, y_{2}>0$ and $y_{3} \neq 0$. Then since $k \geq x_{4}(\mathbf{v})=$ $2 y_{1}+y_{2}(k-1)-y_{3} k$ we find $y_{3}>0$, so all three coefficients are positive. If $y_{1}=y_{2}=y_{3}=1$, then we have $\mathbf{v}=\mathbf{v}_{1}+\mathbf{v}_{2}+\mathbf{v}_{3}=-\mathbf{v}_{4}$. Otherwise $\max \left(y_{1}, y_{2}, y_{3}\right)>1$. If $y_{2}>y_{1}$ then $x_{5}(\mathbf{v})=-y_{1}+y_{2}+y_{3}(k-1) \geq 1+k-1=k$ and then $\left|\mathbf{v}^{+}\right| \geq x_{1}(\mathbf{v})+x_{5}(\mathbf{v}) \geq 1+k>k$, a contradiction, hence $y_{2} \leq y_{1}$. Similarly if $y_{3}=\max \left(y_{1}, y_{2}, y_{3}\right)>1$, then $x_{5}(\mathbf{v})=-y_{1}+y_{2}+y_{3}(k-1) \geq$ $-y_{3}+1+y_{3}(k-1)=y_{3}(k-2)+1 \geq 2(k-2)+1=2 k-3>k$, a contradiction, and hence we may suppose that $y_{1}=\max \left(y_{1}, y_{2}, y_{3}\right)>1$. Now this implies $-k \leq x_{2}(\mathbf{v})=-y_{1}(k-1)+y_{2} \leq-y_{1}(k-1)+y_{1}=(2-k) y_{1} \leq(2-k) 2=4-2 k$, and therefore $k \geq 4$. In fact equality must occur, so $y_{1}=y_{2}=2$, but then $\mathbf{v}=\left(2,-4,-6+y_{3}, 8-4 y_{3}, 3 y_{3}\right)$, and requiring $x_{3}(\mathbf{v}) \geq-k$ and $x_{5}(\mathbf{v}) \leq k$ forces the contradictory inequalities $y_{3} \geq 2$ and $y_{3} \leq 1$.

As these five cases cover all possibilities, the proof is complete.

By Theorem ??, there exists an almost representable order on $\mathcal{P}_{k}[5]$ which fails $C_{4}$, and by Proposition ?? it follows that $g(5, k)=4$ for all $k \geq 4$.

\subsection{Proof of Theorem ?? for $n \geq 6$ and $k \geq 3$.}

The proof for this case is split into a number of observations and lemmata. Let us consider the following $(n-2) \times n$ matrix:

$$
A=\left[\begin{array}{ccccccccccc}
1 & -(k-1) & k-3 & 1 & 0 & \ldots & 0 & 0 & 0 & 1 & -1 \\
0 & 1 & -k & k-2 & 1 & 0 & \ldots & 0 & 0 & 0 & 0 \\
0 & 0 & 1 & -k & k-2 & 1 & 0 & \ldots & 0 & 0 & 0 \\
0 & 0 & 0 & 1 & -k & k-2 & 1 & 0 & \ldots & 0 & 0 \\
\ldots & \ldots & \ldots & \ldots & \ldots & \ldots & \ldots & \ldots & \ldots & \ldots & \ldots \\
\ldots & \ldots & \ldots & \ldots & \ldots & \ldots & \ldots & \ldots & \ldots & \ldots & \ldots \\
0 & 0 & 0 & 0 & 0 & \ldots & 0 & 1 & -k & k-2 & 1 \\
0 & 0 & 0 & 0 & 0 & 0 & \ldots & 0 & 1 & -k & k-1
\end{array}\right]
$$

Let $\mathbf{v}_{i}$ be the $i$ th row of $A$. Clearly the vectors $\mathbf{v}_{1}, \mathbf{v}_{1}, \ldots, \mathbf{v}_{n-2}$ are linearly independent, and therefore span a subspace of $\mathbb{R}^{n}$ of dimension $n-2$. Also it is easy to check that $\left|\mathbf{v}_{i}^{+}\right|=\left|\mathbf{v}_{i}^{-}\right|=k$ for all $i$, and that $\left|\max \left(-\mathbf{v}_{i}^{-}, \mathbf{v}_{j}^{+}\right)\right|>k$ whenever $i \neq j$. 
Let $\mathbf{v}=\sum_{i=1}^{n-2} y_{i} \mathbf{v}_{i}$ be a linear combination of $\mathbf{v}_{1}, \ldots, \mathbf{v}_{n-2}$. For later use, we note the following:

$$
\begin{aligned}
x_{1}(\mathbf{v}) & =y_{1}, \\
x_{2}(\mathbf{v}) & =-y_{1}(k-1)+y_{2}, \\
x_{3}(\mathbf{v}) & =y_{1}(k-3)-y_{2} k+y_{3}, \\
x_{i}(\mathbf{v}) & =y_{i-3}+y_{i-2}(k-2)-y_{i-1} k+y_{i} \text { for } 4 \leq i \leq n-2, \\
x_{n-1}(\mathbf{v}) & =y_{1}+y_{n-4}+y_{n-3}(k-2)-y_{n-2} k, \text { and } \\
x_{n}(\mathbf{v}) & =-y_{1}+y_{n-3}+y_{n-2}(k-1) .
\end{aligned}
$$

In addition, we will use particular sums of these co-ordinates, including

$$
\begin{aligned}
x_{1}(\mathbf{v})+x_{3}(\mathbf{v}) & =y_{1}(k-2)-y_{2} k+y_{3}, \\
x_{2}(\mathbf{v})+x_{3}(\mathbf{v}) & =-2 y_{1}-y_{2}(k-1)+y_{3}, \\
x_{1}(\mathbf{v})+x_{3}(\mathbf{v})+x_{4}(\mathbf{v}) & =y_{1}(k-1)-2 y_{2}-y_{3}(k-1)+y_{4},
\end{aligned}
$$

and, for $4 \leq i \leq n-2$, also the sums

$$
\begin{gathered}
x_{i}(\mathbf{v})+x_{i+1}(\mathbf{v})=y_{i-3}+y_{i-2}(k-1)-2 y_{i-1}-y_{i}(k-1)+y_{i+1}, \\
x_{2}(\mathbf{v})+x_{3}(\mathbf{v})+x_{i}(\mathbf{v})=-2 y_{1}-y_{2}(k-1)+y_{3}+y_{i-3}+y_{i-2}(k-2)-y_{i-1} k+y_{i}, \\
x_{2}(\mathbf{v})+x_{3}(\mathbf{v})+\ldots+x_{i}(\mathbf{v})=-y_{1}-y_{i-2}-y_{i-1}(k-1)+y_{i} .
\end{gathered}
$$

All of these sums must lie in the interval $M_{k}=\{-k, \ldots,-1,0,1, \ldots, k\}$, irrespective of whether their terms $x_{j}(\mathbf{v})$ are positive or negative (since, for example, if the sum exceeds $k$ then the sub-sum of positive terms exceeds $k$ ).

Lemma 3. Suppose that some linear combination $\mathbf{v}=\sum_{i=1}^{n-2} y_{i} \mathbf{v}_{i}$ of the $\mathbf{v}_{i}$ belongs to $T_{k}^{n}$. Then $y_{i} \in \mathbb{Z}$ for all $i$.

Proof. We have $y_{1}=x_{1}(\mathbf{v}) \in \mathbb{Z}$, and as $-y_{1}(k-1)+y_{2}=x_{2}(\mathbf{v}) \in \mathbb{Z}$ we find $y_{2} \in \mathbb{Z}$. Clearly this argument can be repeated, to show $y_{i} \in \mathbb{Z}$ for all $i$.

Lemma 4. There is a vector $\mathbf{w}=\left(w_{1}, \ldots, w_{n}\right)$ belonging to the null space of $A$ such that $w_{1}>w_{2}>\ldots>w_{n}>0$.

Proof. The null space of $A$ has dimension 2 ( since $\mathbf{v}_{1}, \ldots, \mathbf{v}_{n-2}$ are linearly independent), and contains the vector $\mathbf{n}=(1,1, \ldots, 1)$. To construct another vector $\mathbf{u}=\left(u_{1}, \ldots, u_{n}\right)$ in this null space, let us set $u_{n}=0$ and $u_{n-1}=1$. Then from orthogonality with the last row of $A$ we see that $u_{n-2}=k$. We proceed by reverse induction to prove that $u_{i}>2 u_{i+1}$ for $2 \leq i \leq n-1$. This is clearly true for $i=n-1$, and for $i=n-2$ since $k>2$. Now let 
us assume that $u_{i+1} \geq 2 u_{i+2}$ and $u_{i+2} \geq 2 u_{i+3}$ for some $i \leq n-3$. Then by orthogonality with the $i$ th row of $A$ we have the inductive step

$$
\begin{aligned}
u_{i} & =k u_{i+1}-(k-2) u_{i+2}-u_{i+3} \\
& =2 u_{i+1}+(k-2)\left(u_{i+1}-u_{i+2}\right)-u_{i+3} \\
& \geq 2 u_{i+1}+(k-2) u_{i+2}-u_{i+3} \\
& \geq 2 u_{i+1}+u_{i+2}-u_{i+3} \\
& \geq 2 u_{i+1},
\end{aligned}
$$

as required. Similarly, orthogonality with the first row of $A$ gives

$$
\begin{aligned}
u_{1} & =(k-1) u_{2}-(k-3) u_{3}-u_{4}=2 u_{2}+(k-3)\left(u_{2}-u_{3}\right)-u_{4} \\
& \geq 2 u_{2}+(k-3) u_{3}-u_{4} \geq 2 u_{2}-u_{4}>u_{2}+u_{3}-u_{4} \geq u_{2} .
\end{aligned}
$$

Thus we have $u_{1}>u_{2}>\ldots>u_{n}=0$, and we can simply take $\mathbf{w}=\mathbf{u}+\mathbf{n}$ to satisfy the requirement that all co-ordinates of $\mathbf{w}$ are positive.

Lemma 5. $L_{\mathbf{w}} \cap T_{k}^{n}=\left\{\mathbf{0}, \pm \mathbf{v}_{1}, \pm \mathbf{v}_{2}, \ldots, \pm \mathbf{v}_{n-2}, \pm \mathbf{v}_{n-1}\right\}$, where $\mathbf{v}_{n-1}$ is the vector $-\left(\sum_{i=1}^{n-2} \mathbf{v}_{i}\right)=(-1, k-2,2,0, \ldots, 0,-k+1)$.

Proof. Clearly $\left\{\mathbf{v}_{1}, \mathbf{v}_{2}, \ldots, \mathbf{v}_{n-2}\right\}$ is a basis for the $(n-2)$-dimensional subspace $L_{\mathbf{w}} \cap L_{\mathbf{n}}$, so every vector $\mathbf{v}$ in $L_{\mathbf{w}} \cap T_{k}^{n}$ is of the form $\mathbf{v}=\sum_{i=1}^{n-2} y_{i} \mathbf{v}_{i}$, and by Lemma ??, all the $y_{i}$ are integers. To find all possibilities for $\mathbf{v}$, we may assume without loss of generality that $y_{1} \geq 0\left(\right.$ since $\left.-T_{k}^{n}=T_{k}^{n}\right)$. As before, we consider several cases:

Case 1: Suppose $y_{1}=0$. If $y_{i}=0$ for all $i$ then $\mathbf{v}=\mathbf{0}$. Otherwise let $j$ be the smallest positive integer for which $y_{j} \neq 0$. Then without loss of generality we may suppose that $y_{j} \geq 1$. With these hypotheses, we will prove that $\mathbf{v}$ is one of $\mathbf{v}_{2}, \mathbf{v}_{3}, \ldots, \mathbf{v}_{n-3}$ or $\mathbf{v}_{n-2}$. To do so, assume the contrary, and further, suppose that $\sum_{i=2}^{n-2}\left|y_{i}\right|$ is as small as possible.

Now assume also for the time being that $y_{j} \geq 2$. If $j=n-2$, then $x_{n}(\mathbf{v})=-y_{1}+y_{n-3}+y_{n-2}(k-1)=0+0+y_{n-2}(k-1) \geq 2(k-1)>k, \mathrm{a}$ contradiction, and so $j \leq n-3$. The requirement $x_{j+1}(\mathbf{v}) \geq-k$ then gives $y_{j+1} \geq y_{j} k-k \geq 2 k-k=k \geq 3$. But now if $y_{\ell} \geq 3$ and $y_{i} \geq 2$ whenever $j \leq i<\ell$, where $3 \leq \ell \leq n-3$, then $x_{2}(\mathbf{v})+x_{3}(\mathbf{v})+\ldots+x_{\ell+1}(\mathbf{v}) \geq-k$ gives $y_{\ell+1} \geq-k+y_{1}+y_{\ell-1}+y_{\ell}(k-1) \geq-k+0+2+3(k-1)=2 k-1 \geq 3$, and hence by induction, $y_{\ell} \geq 3$ whenever $j<\ell \leq n-2$. This, however, gives

$$
x_{n}(\mathbf{v})=-y_{1}+y_{n-3}+y_{n-2}(k-1) \geq 0+2+3(k-1)=3 k-1>k,
$$


another contradiction. Hence $y_{j}=1$. Also $j \neq n-2$, since we have assumed $\mathbf{v} \neq \mathbf{v}_{n-2}$, and therefore $2 \leq j \leq n-3$.

Next, because $x_{j+1}(\mathbf{v}) \geq-k$ we have $y_{j+1} \geq y_{j} k-k \geq k-k=0$. Assume for the time being that $y_{j+1} \geq 2$. If $j=n-3$, then we find $x_{n}(\mathbf{v})=-y_{1}+y_{n-3}+y_{n-2}(k-1)=0+1+y_{n-2}(k-1) \geq 1+2(k-1)>k$, a contradiction, and so $j \leq n-4$. Moreover, from $x_{j+1}(\mathbf{v})+x_{j+2}(\mathbf{v}) \geq-k$ we find that $y_{j+2} \geq 2 y_{j}+y_{j+1}(k-1)-k \geq 2+2(k-1)-k=k \geq 3$, and by induction (as above), it follows that $y_{\ell} \geq 3$ whenever $j+1<\ell \leq n-2$, and again $x_{n}(\mathbf{v})=-y_{1}+y_{n-3}+y_{n-2}(k-1) \geq 0+2+3(k-1)=3 k-1>k$, a contradiction. Hence $y_{j+1}=0$ or 1 .

If $y_{j+1}=0$, then $x_{j}(\mathbf{v})=x_{j}\left(\mathbf{v}_{j}\right)=1$ and $x_{j+1}(\mathbf{v})=x_{j+1}\left(\mathbf{v}_{j}\right)=-k$, and these co-ordinates cancel each other when we take the difference $\mathbf{u}=\mathbf{v}-\mathbf{v}_{j}$. Moreover, as $|\mathbf{v}| \leq 2 k$ and $\left|\mathbf{v}_{j}\right|=2 k$ we see that $|\mathbf{u}|=\left|\mathbf{v}-\mathbf{v}_{j}\right| \leq(2 k-(k+$ $1))+(2 k-(k+1))<2 k$, and since $\mathbf{u}$ lies in $L_{\mathbf{w}}$ (so that $\left|\mathbf{u}^{+}\right|=\left|\mathbf{u}^{-}\right|$), it follows that $\mathbf{u}$ lies in $T_{k}^{n}$, and hence also in $L_{\mathbf{w}} \cap T_{k}^{n}$. But $\mathbf{u}=\mathbf{v}-\mathbf{v}_{j}=\sum_{i=j+2}^{n-2} y_{i} \mathbf{v}_{i}$, and as $\sum_{i=j+2}^{n-2}\left|y_{i}\right|$ is smaller than $\sum_{i=2}^{n-2}\left|y_{i}\right|$ we deduce that $\mathbf{u}=\mathbf{0}$ or $\mathbf{u}= \pm \mathbf{v}_{i}$ for some $i \geq j+2$, and hence $\mathbf{v}=\mathbf{v}_{j}$ or $\mathbf{v}_{j} \pm \mathbf{v}_{i}$ for some $i \geq j+2$. The former possibility has been ruled out already, and the latter possibility is easily ruled out since $x_{j+1}\left(\mathbf{v}_{j}+\mathbf{v}_{i}\right)+x_{i+1}\left(\mathbf{v}_{j}+\mathbf{v}_{i}\right) \leq-k+1-k=1-2 k<-k$ and $x_{j+1}\left(\mathbf{v}_{j}-\mathbf{v}_{i}\right)+x_{i+2}\left(\mathbf{v}_{j}-\mathbf{v}_{i}\right) \leq-k-(k-2)=2-2 k<-k$.

Similarly if $y_{j+1}=1$, then $x_{j}(\mathbf{v})=x_{j}\left(\mathbf{v}_{j}\right)=1$ while $x_{j+1}(\mathbf{v})=-(k-1)$ and $x_{j+1}\left(\mathbf{v}_{j}\right)=-k$, so that when we take the difference $\mathbf{u}=\mathbf{v}-\mathbf{v}_{j}$, we find $|\mathbf{u}|=\left|\mathbf{v}-\mathbf{v}_{j}\right| \leq(2 k-k)+(2 k-k)=2 k$, and again $\mathbf{u}$ lies in $L_{\mathbf{w}} \cap T_{k}^{n}$, but $\mathbf{u}=\mathbf{v}-\mathbf{v}_{j}=\sum_{i=j+1}^{n-2} y_{i} \mathbf{v}_{i}$, with $\sum_{i=j+1}^{n-2}\left|y_{i}\right|$ smaller than $\sum_{i=2}^{n-2}\left|y_{i}\right|$, so $\mathbf{u}=\mathbf{0}$ or $\mathbf{u}= \pm \mathbf{v}_{i}$ for some $i \geq j+1$, and hence $\mathbf{v}=\mathbf{v}_{j}$ or $\mathbf{v}_{j} \pm \mathbf{v}_{i}$ for some $i \geq j+1$. In fact because $y_{j+1}=1$ we must have $\mathbf{v}=\mathbf{v}_{j}+\mathbf{v}_{j+1}$, but then $\left.x_{j}(\mathbf{v})+x_{j+3}(\mathbf{v})+x_{j+4}(\mathbf{v})\right)=1+(k-1)+1=k+1>k$, another contradiction.

Hence there is no other such $\mathbf{v}$; the only possibilities are $\mathbf{v}_{2}, \mathbf{v}_{3}, \ldots, \mathbf{v}_{n-3}$ and $\mathbf{v}_{n-2}$.

Case 2: Suppose $y_{1}=1$ and $y_{2}<0$. Then the requirement $x_{2}(\mathbf{v}) \geq-k$ gives $y_{2}=-1$ and $x_{2}(\mathbf{v})=-k$, so that no other co-ordinates of $\mathbf{v}$ can be negative. Similarly, from $x_{1}(\mathbf{v})+x_{3}(\mathbf{v}) \leq k$ we find that $y_{3} \leq k-y_{1}(k-2)+y_{2} k \leq$ $k-(k-2)-k=2-k \leq-1$, and from $x_{1}(\mathbf{v})+x_{3}(\mathbf{v})+x_{4}(\mathbf{v}) \leq k$ we find that $y_{4} \leq k-y_{1}(k-1)+2 y_{2}+(k-1) y_{3} \leq k-(k-1)-2-(k-1)=$ $-k \leq-3$. It now follows by induction that $y_{j} \leq-3$ for $4 \leq j \leq n-2$, for if $y_{j} \leq-3$ and $y_{i} \leq-1$ for $2 \leq i<j$, then from the requirement $x_{2}(\mathbf{v})+x_{3}(\mathbf{v})+\ldots+x_{j+1}(\mathbf{v}) \geq-k$ we find that

$y_{j+1} \leq k+y_{1}+y_{j-1}+y_{j}(k-1) \leq k+1-1-3(k-1) \leq 3-2 k \leq-3$. 
This, however, gives

$$
x_{n}(\mathbf{v})=-y_{1}+y_{n-3}+y_{n-2}(k-1) \leq-1-1-3(k-1)=1-3 k<-k,
$$

a contradiction. Hence this case is impossible.

Case 3: Suppose $y_{1}=1$ and $y_{2}=0$. As $\mathbf{v}$ and $\mathbf{v}_{1}$ then have the same first two co-ordinates, namely 1 and $-(k-1)$, these cancel each other when taking their difference $\mathbf{u}=\mathbf{v}-\mathbf{v}_{1}$. Moreover, as $|\mathbf{v}| \leq 2 k$ and $\left|\mathbf{v}_{1}\right|=2 k$ we see that $|\mathbf{u}|=\left|\mathbf{v}-\mathbf{v}_{1}\right| \leq(2 k-k)+(2 k-k)=2 k$, and since $\mathbf{u}$ lies in $L_{\mathbf{w}}$ (so that $\left|\mathbf{u}^{+}\right|=\left|\mathbf{u}^{-}\right|$), it follows that $\mathbf{u}$ lies in $T_{k}^{n}$. By the argument in Case 1 , we find that $\mathbf{u}=\mathbf{0}$ or $\pm \mathbf{v}_{i}$ for some $i \geq 3$, and hence $\mathbf{v}=\mathbf{v}_{1}$ or $\mathbf{v}_{1} \pm \mathbf{v}_{i}$ for some $i \geq 3$. The latter possibility, however, is easily ruled out since $x_{2}\left(\mathbf{v}_{1}+\mathbf{v}_{i}\right)+x_{i+1}\left(\mathbf{v}_{1}+\mathbf{v}_{i}\right) \leq-(k-1)+1-k=2-2 k<-k$ and $x_{1}\left(\mathbf{v}_{1}-\mathbf{v}_{i}\right)+x_{i+1}\left(\mathbf{v}_{1}-\mathbf{v}_{i}\right) \geq 1+k>k$. Hence we have only $\mathbf{v}=\mathbf{v}_{1}$.

Case 4: Suppose $y_{1}=1$ and $y_{2}>0$. Assuming $y_{2} \geq 2$, then the requirement $x_{2}(\mathbf{v})+x_{3}(\mathbf{v}) \geq-k$ gives $y_{3} \geq 2 y_{1}+y_{2}(k-1)-k \geq 2+2(k-1)-k=k \geq 3$. It then follows by induction that $y_{j} \geq 3$ for $4 \leq j \leq n-2$, for if $y_{i} \geq 3$ for $3 \leq i \leq j-1$, then from

$x_{2}(\mathbf{v})+x_{3}(\mathbf{v})+\ldots+x_{j}(\mathbf{v}) \geq-k$ we find that

$$
y_{j} \geq-k+y_{1}+y_{j-2}+y_{j-1}(k-1) \geq-k+1+2+3(k-1) \geq 2 k \geq 3 \text {. }
$$

This, however, gives

$$
x_{n}(\mathbf{v})=-y_{1}+y_{n-3}+y_{n-2}(k-1) \geq-1+3+3(k-1)=3 k-1>k,
$$

a contradiction. Thus $y_{2}=1$.

If all $y_{i}=1$ for all $i$ then $\mathbf{v}=\sum_{i=1}^{n-2} \mathbf{v}_{i}=-\mathbf{v}_{n-1}$. Otherwise suppose that $j$ is the smallest positive integer for which $y_{j} \neq 1$. If $j=3$, then from $x_{2}(\mathbf{v})+x_{3}(\mathbf{v}) \geq-k$ we find that $y_{3} \geq 2 y_{1}+y_{2}(k-1)-k=1$, and so $y_{j} \geq 2$ in that case, while if $3 \leq j \leq n-2$ then $x_{2}(\mathbf{v})+x_{3}(\mathbf{v})+x_{j}(\mathbf{v}) \geq-k$ gives

$$
\begin{aligned}
y_{j} & \geq-k+2 y_{1}+y_{2}(k-1)-y_{3}-y_{j-3}-y_{j-2}(k-2)+y_{j-1} k \\
& =-k+2+(k-1)-1-1-(k-2)+k=1,
\end{aligned}
$$

and so $y_{j} \geq 2$ in that case also. But now if $y_{\ell} \geq 2$ and $y_{i} \geq 1$ for all $i<\ell$, where $3 \leq \ell \leq n-3$, then $x_{2}(\mathbf{v})+x_{3}(\mathbf{v})+\ldots+x_{\ell+1}(\mathbf{v}) \geq-k$ gives

$$
y_{\ell+1} \geq-k+y_{1}+y_{\ell-1}+y_{\ell}(k-1) \geq-k+1+1+2(k-1) \geq k \geq 3 \text {, }
$$

and hence by induction, $y_{\ell} \geq 2$ whenever $j \leq \ell \leq n-2$. This, however, gives

$$
x_{n}(\mathbf{v})=-y_{1}+y_{n-3}+y_{n-2}(k-1) \geq-1+1+2(k-1)>k,
$$


a contradiction. Hence no such $j$ exists, and we have only $\mathbf{v}=-\mathbf{v}_{n-1}$.

Case 5: Suppose $y_{1} \geq 2$. Then since $x_{2}(\mathbf{v}) \geq-k$ we have $y_{2} \geq y_{1}(k-1)-k=$ $\left(y_{1}-1\right)(k-1)-1 \geq 2\left(y_{1}-1\right)-1=2 y_{1}-3 \geq 1$, and since $x_{2}(\mathbf{v})+x_{3}(\mathbf{v}) \geq-k$ we have $y_{3} \geq 2 y_{1}+y_{2}(k-1)-k=2 y_{1}+\left(y_{2}-1\right)(k-1)-1 \geq 2 y_{1}-1 \geq y_{1}+1 \geq 3$. It follows by induction that $y_{j} \geq y_{1}+1 \geq 3$ for $4 \leq j \leq n-2$, for if $y_{i} \geq y_{1}+1 \geq 3$ for $3 \leq i \leq j-1$, then from $x_{2}(\mathbf{v})+x_{3}(\mathbf{v})+\ldots+x_{j}(\mathbf{v}) \geq-k$ we find that

$$
\begin{aligned}
y_{j} & \geq-k+y_{1}+y_{j-2}+y_{j-1}(k-1) \geq-k+y_{1}+1+3(k-1) \\
& \geq y_{1}+2(k-1) \geq y_{1}+1 \geq 3 .
\end{aligned}
$$

This, however, gives

$$
x_{n}(\mathbf{v})=-y_{1}+y_{n-3}+y_{n-2}(k-1)>1+3(k-1)=3 k-2>k,
$$

a contradiction.

As these five cases cover all possibilities, the proof is complete.

\subsection{Proof of Theorem ?? for $n \geq 8$ and $k=2$.}

Consider the $n-2$ vectors $\mathbf{v}_{1}, \ldots, \mathbf{v}_{n-2}$ that make up the rows of the following $(n-2) \times n$ matrix:

$$
A=\left[\begin{array}{rrrrrrrrrrrrrr}
-1 & 1 & 0 & 1 & 0 & 0 & 0 & 0 & \ldots & 0 & 0 & 0 & 0 & -1 \\
1 & 0 & -2 & 0 & 0 & 0 & 0 & 0 & \ldots & 0 & 0 & 0 & 1 & 0 \\
0 & 0 & 1 & -2 & 0 & 1 & 0 & 0 & \ldots & 0 & 0 & 0 & 0 & 0 \\
0 & 0 & 0 & 1 & -2 & 0 & 1 & 0 & \ldots & 0 & 0 & 0 & 0 & 0 \\
0 & 0 & 0 & 0 & 1 & -2 & 0 & 1 & \ldots & 0 & 0 & 0 & 0 & 0 \\
\ldots & \ldots & \ldots & \ldots & \ldots & \ldots & \ldots & \ldots & \ldots & \ldots & \ldots & \ldots & \ldots & \ldots \\
0 & 0 & 0 & 0 & 0 & 0 & 0 & 0 & \ldots & 1 & -2 & 0 & 1 & 0 \\
0 & 0 & 0 & 0 & 0 & 0 & 0 & 0 & \ldots & 0 & 1 & -2 & 0 & 1 \\
0 & 0 & 0 & 0 & 0 & 0 & 0 & 0 & \ldots & 0 & 0 & 1 & -2 & 1
\end{array}\right] .
$$

Clearly these vectors are linearly independent, and so the row-space of $A$ a subspace of $\mathbb{R}^{n}$ of dimension $n-2$. Also clearly $\left|\mathbf{v}_{i}^{+}\right|=\left|\mathbf{v}_{i}^{-}\right|=2$ for all $i$, and it is easy to check that $\left|\max \left(-\mathbf{v}_{i}^{-}, \mathbf{v}_{j}^{+}\right)\right|>2$ whenever $i \neq j$.

In what follows, we use the Fibonacci sequence, that is, the sequence $\left(f_{m}\right)$ of integers defined by $f_{1}=f_{2}=1$ and $f_{m+2}=f_{m+1}+f_{m}$ for all $m \geq 1$.

Lemma 6. There is a vector $\mathbf{w}=\left(w_{1}, \ldots, w_{n}\right)$ belonging to the null space of $A$ such that $w_{1}>w_{2}>\ldots>w_{n}>0$. In fact, such a vector is given by

$$
w_{1}=2 f_{n-1}-2, \quad w_{2}=2 f_{n-1}-f_{n-2}-1, \text { and } w_{i}=f_{n-i+2}
$$

for $3 \leq i \leq n$, where $f_{m}$ is the $m$ th term of the Fibonacci sequence. 
Proof. Let us take $w_{n}=f_{2}=1$ and $w_{n-1}=f_{3}=2$. Then by orthogonality with $\mathbf{v}_{n-2}$ we have $w_{n-2}=2 w_{n-2}-w_{n}=3=f_{4}$. It follows by induction that $w_{i}=f_{n-i+2}$ whenever $3 \leq i \leq n$, for if this is true for all $j$ such that $i<j \leq n$, then by orthogonality with $\mathbf{v}_{i}$ we have

$$
\begin{aligned}
w_{i} & =2 w_{i+1}-w_{i+3}=2 f_{n-(i+1)+2}-f_{n-(i+3)+2} \\
& =2 f_{n-i+1}-\left(f_{n-i+1}-f_{n-i}\right)=f_{n-i+1}+f_{n-i} \\
& =f_{n-i+2} .
\end{aligned}
$$

Next, by orthogonality with $\mathbf{v}_{2}$ we have $w_{1}=2 w_{3}-w_{n-1}=2 f_{n-1}-2$, and by orthogonality with $\mathbf{v}_{1}$ we have $w_{2}=w_{1}-w_{4}+w_{n}=2 f_{n-1}-2-f_{n-2}+1=$ $2 f_{n-1}-f_{n-2}-1=f_{n-1}+f_{n-3}-1$. Finally, it is an easy exercise to verify that $w_{1}>w_{2}>\ldots>w_{n}>0$, because $\left(f_{m}\right)$ is an increasing sequence of positive integers.

Lemma 7. $L_{\mathbf{w}} \cap T_{2}^{n}=\left\{\mathbf{0}, \pm \mathbf{v}_{1}, \pm \mathbf{v}_{2}, \ldots, \pm \mathbf{v}_{n-2}, \pm \mathbf{v}_{n-1}\right\}$, where $\mathbf{v}_{n-1}$ is the vector $-\left(\sum_{i=1}^{n-2} \mathbf{v}_{i}\right)=(0,1,-1,0,-1,0, \ldots, 0,1)$.

Proof. Let $\mathbf{v}$ be any non-zero vector in $L_{\mathbf{w}} \cap T_{2}^{n}$, and let $i$ be the smallest positive integer such that $x_{i}(\mathbf{v}) \neq 0$. Without loss of generality we may assume that $x_{i}(\mathbf{v})>0$, and therefore $x_{i}(\mathbf{v})=1$ or 2 . If $x_{i}(\mathbf{v})=2$, however, then orthogonality of $\mathbf{v}$ with $\mathbf{w}$ must correspond to an equation of the form $2 w_{i}-w_{r}-w_{s}=0$ with $i<r<s$ or $2 w_{i}-2 w_{r}$ with $i<r$, both of which are impossible since the co-ordinates of $\mathbf{w}$ are strictly decreasing. Thus $x_{i}(\mathbf{v})=1$, and orthogonality of $\mathbf{v}$ with $\mathbf{w}$ must correspond to an equation of the form $w_{i}-w_{r}-w_{s}+w_{t}$ with $i<r<s<t$, or $w_{i}-2 w_{r}+w_{t}=0$ with $i<r<t$. These two possibilities can be amalgamated into one by considering the equation as $w_{i}-w_{r}-w_{s}+w_{t}$ with $i<r \leq s<t$. Again we proceed case-by-case.

Case 1: Suppose $i=1$. Then $w_{1}=w_{r}+w_{s}-w_{t}<2 w_{r}$ so $2 f_{n-1}-2<2 f_{n-r+2}$ and therefore $f_{n-1}<f_{n-r+2}+1$, which implies $r \leq 3$. Next, if $r=3$ then $w_{1}=w_{3}+w_{s}-w_{t}<w_{3}+w_{s}$, so $2 f_{n-1}-2<f_{n-1}+f_{n-s+2}$ and therefore $f_{n-s+2}>f_{n-1}-2$, which implies $s \leq 3$ (because $n \geq 8$ ), giving $r=s=3$ and then $w_{t}=2 w_{3}-w_{1}=2 f_{n-1}-2 f_{n-1}+2=2$, so $t=n-1$ and $\mathbf{v}=(1,0,-2,0, \ldots, 0,1,0)=\mathbf{v}_{2}$. Similarly, if $r=2$ then we find that $w_{1}=w_{2}+w_{s}-w_{t}<w_{2}+w_{s}$, so $2 f_{n-1}-2<2 f_{n-1}-f_{n-2}-1+f_{n-s+2}$ and therefore $f_{n-s+2}>f_{n-2}-1$, which implies $s \leq 4$. If $r=s=2$, however, then $w_{t}=2 w_{2}-w_{1}=2\left(2 f_{n-1}-f_{n-2}-1\right)-2 f_{n-1}+2=2 f_{n-1}-2 f_{n-2}=2 f_{n-3}$, which is impossible (since $2 f_{n-3}>f_{n-3}+f_{n-4}=f_{n-2}$ while also $2 f_{n-3}<$ $\left.f_{n-3}+f_{n-2}=f_{n-1}\right)$. Similarly, if $r=2$ and $s=3$ then $w_{t}=w_{2}+w_{3}-w_{1}=$ $2 f_{n-1}-f_{n-2}-1+f_{n-1}-2 f_{n-1}+2=f_{n-1}-f_{n-2}+1=f_{n-3}+1$, which is also impossible (since $n-3 \geq 5$ ). Hence if $r=2$ then $s=4$, in which case 
$w_{t}=w_{2}+w_{4}-w_{1}=2 f_{n-1}-f_{n-2}-1+f_{n-2}-2 f_{n-1}+2=1$, giving $t=n$ and $\mathbf{v}=(1,-1,0,-1,0, \ldots, 0,1)=-\mathbf{v}_{1}$.

Case 2: Suppose $i=2$. Then $2 w_{r}>w_{r}+w_{s}-w_{t}=w_{2}$ so

$$
\begin{aligned}
2 f_{n-r+2} & >2 f_{n-1}-f_{n-2}-1=2\left(f_{n-2}+f_{n-3}\right)-f_{n-2}-1 \\
& =f_{n-2}+2 f_{n-3}-1>f_{n-2}+f_{n-3}+f_{n-4}=2 f_{n-2},
\end{aligned}
$$

and therefore $r \leq 3$, so $r=3$, and

$$
w_{s}-w_{t}=w_{2}-w_{3}=f_{n-1}-f_{n-2}-1=f_{n-3}-1 .
$$

If $s \geq 6$, then $n-s+2<n-3$ and so $w_{s}-w_{t}=f_{n-s+2}-f_{n-t+2}<f_{n-3}-1$, a contradiction, hence $3 \leq s \leq 5$. If $s=5$, then $f_{n-3}-1=w_{s}-w_{t}=$ $f_{n-s+2}-f_{n-t+2}=f_{n-3}-f_{n-t+2}$ so $f_{n-t+2}=1$, giving $t=n$, and then $\mathbf{v}=(0,1,-1,0,-1,0, \ldots, 0,1)=\mathbf{v}_{n-1}$. If $s=4$, then $f_{n-t+2}=w_{t}=$ $w_{s}-f_{n-3}+1=f_{n-2}-f_{n-3}+1=f_{n-4}+1$, which is impossible since $n \geq 8$. Finally (in the case where $i=2$ ) if $s=3$, then $t \geq 4$ so $w_{t} \leq f_{n-2}$ and therefore $w_{s}-w_{t} \geq f_{n-1}-f_{n-2}=f_{n-3}>f_{n-3}-1$, another contradiction.

Case 3: Suppose $i \geq 3$. If $r \geq i+2$, then $f_{n-s+2}=w_{s}>w_{s}-w_{t}=w_{i}-w_{r} \geq$ $w_{i}-w_{i+2}=f_{n-i+2}-f_{n-i}=f_{n-i+1}$, which implies $n-s+2>n-i+1$, so $s<i+1<r$, a contradiction. Thus $r=i+1$, and $w_{s}-w_{t}=w_{i}-w_{r}=$ $f_{n-i+2}-f_{n-i+1}=f_{n-i}$. In particular, $f_{n-s+2}=w_{s}>w_{s}-w_{t}=f_{n-i}$, so $n-s+2>n-i$, giving $s<i+2$, and therefore $r=s=i+1$. It follows that $f_{n-t+2}=w_{t}=w_{r}+w_{s}-w_{i}=2 w_{i+1}-w_{i}=2 f_{n-i+1}-f_{n-i+2}=$ $f_{n-i+1}+\left(f_{n-i+1}-f_{n-i+2}\right)=f_{n-i+1}-f_{n-i}=f_{n-i-1}$, and thus either $t=i+3$ (if $i \leq n-3$ ) or $t=i+2=n$ (if $i=n-2$ ), giving $\mathbf{v}=\mathbf{v}_{i}$ in both cases.

Hence the only non-zero vectors in $L_{\mathbf{w}} \cap T_{2}^{n}$ are $\pm \mathbf{v}_{1}, \pm \mathbf{v}_{2}, \ldots, \pm \mathbf{v}_{n-1}$.

By Theorem ??, we have $g(n, 2)=n-1$ for all $n \geq 8$.

\subsection{Remaining cases}

The results for the remaining cases were obtained with the help of the computer algebra system MAGMA [?], using the same techniques of enumeration as described by Fishburn in [?, Section 4], but also checking the results for both representability and almost representability (using techniques of linear programming) as in [?].

In the case where $n=5$ and $k=2$, we have found that there are precisely 286 orders on $\mathcal{P}_{2}[5]$ which satisfy the IES, of which 114 are representable and 172 are not, and that all of the latter fail $C_{3}$, and all but 40 are all almost representable. One example of an almost representable order that fails $C_{3}$ 
is the following (in which ij denotes the obvious multiset of cardinality 2): $1^{2} \succeq 12 \succeq 13 \succeq 14 \succeq 2^{2} \succeq 15 \succeq 23 \succeq 24 \succeq 3^{2} \succeq 34 \succeq 25 \succeq 35 \succeq 4^{2} \succeq$ $45 \succeq 5^{2}$; this is almost representable via the vector $\mathbf{w}=(6,4,3,2,1)$. Thus $f(5,2)=g(5,2)=3$.

For $n=5$ and $k=3$, there are precisely 68820 orders on $\mathcal{P}_{3}[5]$ satisfying the IES, of which 6588 are representable and 62232 are not. Of those which are not representable, 62208 fail $C_{3}$, while 24 satisfy $C_{3}$ but fail $C_{4}$. All of the latter 24 are almost representable, and one such example is given by $1^{3} \succeq 1^{2} 2 \succeq 1^{2} 3 \succeq 1^{2} 4 \succeq 1^{2} 5 \succeq 12^{2} \succeq 123 \succeq 124 \succeq 13^{2} \succeq 125 \succeq 134 \succeq 2^{3} \succeq$ $14^{2} \succeq 135 \succeq 2^{2} 3 \succeq 145 \succeq 2^{2} 4 \succeq 23^{2} \succeq 15^{2} \succeq 2^{2} 5 \succeq 234 \succeq 24^{2} \succeq 3^{3} \succeq 235 \succeq$ $3^{2} 4 \succeq 245 \succeq 34^{2} \succeq 3^{2} 5 \succeq 25^{2} \succeq 4^{3} \succeq 345 \succeq 4^{2} 5 \succeq 35^{2} \succeq 45^{2} \succeq 5^{3}$; this is almost representable via $\mathbf{w}=(20,10,6,4,1)$. Thus $f(5,3)=g(5,3)=4$.

For $n=6$ and $k=2$, there are precisely 33592 orders on $\mathcal{P}_{2}[6]$ which satisfy the IES, of which 2608 are representable and 30984 are not. Of those which are not representable, 30980 fail $C_{3}$, while the other four satisfy $C_{3}$ and $C_{4}$ but fail $C_{5}$. All of the latter four are almost representable, and one such example is $1^{2} \succeq 12 \succeq 13 \succeq 14 \succeq 15 \succeq 2^{2} \succeq 23 \succeq 24 \succeq 3^{2} \succeq 34 \succeq$ $16 \succeq 25 \succeq 4^{2} \succeq 35 \succeq 45 \succeq 26 \succeq 36 \succeq 5^{2} \succeq 46 \succeq 56 \succeq 6^{2}$; this is almost representable via $\mathbf{w}=(12,8,7,6,4,1)$. Thus $f(6,2)=g(6,2)=5$.

For $n=7$ and $k=2$, there are 23178480 orders on $\mathcal{P}_{2}$ [7] which satisfy the IES, of which 107498 are representable and 23070982 are not. Of those which are not representable, 23069816 fail $C_{3}, 1138$ fail $C_{5}$ but satisfy $C_{3}$ and $C_{4}$, and 28 fail $C_{6}$ but satisfy $C_{3}, C_{4}$ and $C_{5}$. Also, of the 1138 that fail $C_{5}$ but satisfy $C_{3}$ and $C_{4}, 1078$ are almost representable, while the other 60 are not almost representable. Finally, all of the 28 that fail $C_{6}$ but satisfy $C_{3}$, $C_{4}$ and $C_{5}$ are almost representable; one such example is $1^{2} \succeq 12 \succeq 13 \succeq$ $14 \succeq 15 \succeq 16 \succeq 2^{2} \succeq 23 \succeq 24 \succeq 25 \succeq 3^{2} \succeq 34 \succeq 17 \succeq 35 \succeq 4^{2} \succeq 45 \succeq 26 \succeq$ $5^{2} \succeq 36 \succeq 46 \succeq 56 \succeq 27 \succeq 37 \succeq 47 \succeq 6^{2} \succeq 57 \succeq 67 \succeq 7^{2}$; this is almost representable via $\mathbf{w}=(22,14,12,11,10,6,1)$. Thus $f(7,2)=g(7,2)=6$.

This completes the proof of Theorem ??. It is interesting to note that for each of the cases $(n, k)=(5,2),(6,2)$ and $(7,2)$, there are no orderings that fail $C_{4}$ but satisfy $C_{3}$. We do not yet understand why that happens.

\section{A Conjecture}

The results of this paper give us reason to formulate the following:

Conjecture 1. $f(n, k)=g(n, k)$ for all $n \geq 3$ and $k \geq 1$. 


\section{References}

[1] Bosma, W., Cannon, J., and Playoust, C.: The Magma Algebra System I: The User Language. J. Symbolic Comput. 24, 235-265 (1997)

[2] Conder, M., Slinko, A.: A Counterexample to Fishburn's Conjecture on Finite Linear Qualitative Probability. Journal of Mathematical Psychology 48(6), 425-431 (2004)

[3] Danilov, V.I. Aggregation of dichotomic preferences, Mathematical Social Sciences 13, 49-58 (1987).

[4] Dershowitz,N. Termination of Rewriting. In: Proc. First Internat. Conf. on Rewriting Techniques and Applications, Lecture Notes in Computer Science, Vol 202 (Springer, Berlin, 180-224 (1985).

[5] de Finnetti, B.: Sul significato soggetivo della probabilità. Fundamenta Mathematicae 17, 298-329 (1931)

[6] Fishburn, P.C.: Finite Linear Qualitative Probability. Journal of Mathematical Psychology. 40, 64-77 (1996)

[7] Fishburn, P.C.: Failure of Cancellation Conditions for Additive Linear Orders. Journal of Combinatorial Design 5, 353-365 (1997)

[8] Herstein, I.N., Milnor J.: An Axiomatic Approach to Measurable Utility. Econometrica 23, 291-297 (1953)

[9] Katzner, W.D.: Static Demand Theory. MacMillan, London (1970)

[10] Koopmans, T.C.: Representation of Preference Orderings with Independent Components of Consumption and Representation of Preference Orderings over Time. in: McGuire, C.B., Radner, R. (Eds.) Decision and Organization. pp. 57-100. North-Holland, Amsterdam, 1972.

[11] Kraft, C.H., Pratt, J.W., Seidenberg, A.: Intuitive Probability on Finite Sets. Annals of Mathematical Statistics 30, 408-419 (1959)

[12] Krantz, D.H., Luce, R.D., Suppes, P., Tversky, A.: Foundations of Measurement, Vol. 1. Academic Press (1971)

[13] Marshak, J.: Rational Behavior, Uncertain Prospects, and Measurable Utility. Econometrica 18, 111-141 (1950)

[14] Martin,U. A Geometric Approach to Multiset Ordering, Theoretical Computer Science, 67, 37-54 (1989). 
[15] Regoli, G.: Comparative probability orderings. In: Imprecise Probabilities Project. Documentation.

http://www.sipta.org/documentation/comparative_prob/regoli.pdf.

Cited 19 Sept 2006 (2006)

[16] Robbiano, L.: Term orderings on the polynomial ring. In: EUROCAL'85, Linz, 1985, vol. 2. Lecture Notes in Comput. Sci., vol. 204, Springer, Berlin, pp. 513-517.

[17] Savage,L.J. The Foundations of Statistics. New York, John Wiley and Sons, 1954.

[18] Sertel, M.R., Kalaycığlu, E.: Toward the Design of a New Electoral Method for Turkey (in Turkish) TÜSIAD, Istanbul (1995)

[19] Sertel, M., Slinko, A.: Ranking Committees, Words or Multisets. Nota di Laboro 50.2002. Center of Operation Research and Economics. The Fundazione Eni Enrico Mattei, Milan (2002)

[20] Sertel, M. and A. Slinko, A.: Ranking Committees, Income Streams or Multisets. Economic Theory, 30(2): 265-287 (2007)

[21] Stanley, R.P.: Enumerative Combinatorics, Vol.1. Cambridge University Press (1997)

[22] von Neumann, J., Morgenstern, O.: Theory of Games and Economic Behavior, Section 3 and Appendix. Princeton University Press (1947)

[23] Wakker, P.P.: Additive Representations of Preferences. Kluwer (1989) 